- News \& Views •

\title{
Advanced Radiative Transfer Modeling System (ARMS): A New-Generation Satellite Observation Operator Developed for Numerical Weather Prediction and Remote Sensing Applications
}

\author{
Fuzhong WENG ${ }^{*}$, Xinwen $\mathrm{YU}^{2}$, Yihong DUAN ${ }^{1}$, Jun $\mathrm{YANG}^{3}$, and Jianjie WANG ${ }^{4}$ \\ ${ }^{1}$ Chinese Academy of Meteorological Sciences, Beijing 100081, China \\ ${ }^{2}$ Chinese Meteorological Administration, Beijing 100081, China \\ ${ }^{3}$ National Meteorological Satellite Center, Beijing 100081, China \\ ${ }^{4}$ National Meteorological Center, Beijing 100081, China
}

(Received 21 August 2019; revised 30 October 2019; accepted 1 November 2019)

\begin{abstract}
Citation: Weng, F. Z., X. W. Yu, Y. H. Duan, J. Yang, and J. J. Wang, 2020: Advanced Radiative Transfer Modeling System (ARMS): A new-generation satellite observation operator developed for numerical weather prediction and remote sensing applications. Adv. Atmos. Sci., 37(2), 131-136, https://doi.org/10.1007/s00376-019-9170-2.
\end{abstract}

\section{Urgent requirements for developing FengYun satellite observation operators}

In the past two decades, satellite measurements have been widely utilized for understanding and predicting weather and climate, and are now an essential component in global observing systems. Atmospheric and surface data observed from satellite, in-situ, and ground-based instruments are assimilated to produce a best representation of the atmospheric state on model grids. The gridded analysis data are derived from the scattered observations and a priori knowledge from the model forecasts. Through the assimilation of satellite data, the global medium-range (7-10 days) forecast score has been significantly improved, especially for the data-void Southern Hemisphere (Le Marshall et al., 2007). Also, the assimilation of satellite data in the Hurricane Weather and Forecast Model improves the prediction of hurricane tracks and intensity, as well as coastal precipitation (Zou et al., 2013).

The data assimilation systems developed at major numerical weather prediction (NWP) centers have undergone more than 50 years of research. Optimal interpolation was initially utilized by NWP centers (until the early 1990s) to assimilate all the observations. Most of the ingested data (>90\%) into numerical models come from satellites, and the highest impacts of satellite data on global medium-range forecasts result from direct assimilation of satellite microwave and infrared sounding radiance data. Since the radiances are not atmospheric-state variables produced by forecast models, a relationship between the state variable and the satellite radiances is required and this relationship is based on the radiative transfer model or observation operator. In addition, the derivative of radiance relative to the state variable or radiance Jacobian is required in satellite data assimilation. Thus, it is important that the observation operator be executed fast and accurately in NWP models in order for a sheer volume of satellite data to be ingested within a limited assimilation time window.

A fast radiative transfer model is effective for assimilation of satellite radiance data into NWP models. The United States and Europe have developed their respective fast models through satellite missions and NWP programs. The Community Radiative Transfer Model (CRTM) was proposed at the US Joint Center for Satellite Data Assimilation in the early 2000s (Weng et al., 2003; Weng, 2007) and has undergone nearly two decades of scientific and software-engineering improvements. The Radiative Transfer for TOVS (RTTOV) model was also developed for simulating satellite radiances at variable wavelengths (Saunders et al., 2018) and is now widely used in the NWP community. In the past, Chinese NWP development has benefited from using RTTOV in its data assimilation systems (Yu et al., 2019). Indeed, both CRTM and RTTOV are performing quite well for NOAA and for European satellite instruments. However, the scientific priority in upgrading CRTM and RTTOV is driven by the United States and European satellite missions. Thus, for the plethora of observation

\footnotetext{
* Corresponding author: Fuzhong WENG

Email: Wengfz@cma.gov.cn
} 
data from Chinese satellites, it is imperative that an independent radiative transfer system be developed to accelerate the use of Chinese satellite data in NWP models and for many other applications. This article describes the ongoing development of such a system, named the Advanced Radiative Transfer Modeling System, or ARMS.

\section{Capabilities of ARMS}

A typical radiative transfer model includes four modular components: (1) atmospheric gaseous absorption; (2) aerosol and cloud absorption and scattering; (3) surface emission and reflection; and (4) radiative transfer schemes. Some of the components may be further split into sub-modules. For example, non-precipitating and precipitating clouds may be treated separately due to their distinct differences in particle size distribution. Surface emissivity and reflectivity modules are developed according to wavelength and surface types, respectively.

Figure 1 illustrates ARMS, whose development is currently underway and is being designed with a modular structure that allows for new "plug and play" software modules. The ARMS forward model computes the emissivity and reflectivity at surfaces, the particle optical parameters, and the radiances at the top of the atmosphere. The ARMS tangent linear module is derived from the forward module, and the adjoint module is then a translation of the tangent linear module. The tangent linear and adjoint modules and their performance evaluations are conducted according to the training materials (Angela Benedetti, 2016, https://earth.esa.int/documents/973910/2642313/AB2b.pdf).

Radiative transfer simulation requires atmospheric optical parameters as inputs. Atmospheric optical thickness varies with atmospheric conditions and is often derived by using a spectroscopic database that has line-by-line (LBL) absorption coefficients. The LBL models are accurate but take a long time to calculate the atmospheric optical thickness profiles. In order to provide accurate optical parameters in the short time allotted in the operational forecast process, we have developed fast models that approximate the LBL calculations for each frequency at the specific satellite viewing angle. In LBLRTM, molecular line absorption parameters are selected from the HITRAN database (Rothman et al, 2013), and the self- and foreign-broadened water vapor continuum model of MT_CKD (Clough et al., 1992) as well as continuum absorption of carbon dioxide are also incorporated. The approach for a fast atmospheric transmittance calculation was developed by McMillin and Fleming (1976), and some incremental changes have been made by the community since then (Saunders et al., 1999; Han et al., 2007). In ARMS, the parameterized optical modules of each satellite instrument are also derived from the LBL calculation and convolved with the instrument spectral response function, resulting in an instrument-specific observation operator. For a hyperspectral IR instrument like CrIS or HIRAS, the accuracy is typically within 0.1 to $0.3 \mathrm{~K}$ within each spectral band.

In ARMS, the optical parameters of five hydrometeors associated with cloud and rain water, cloud ice, snow, and graupel are computed at each model layer. The atmosphere is divided into 100 layers and 101 levels. The liquid-phase hydrometeors are assumed to be spherical in shape and the scattering parameters are derived from Mie theory. The particle size distribution is a modified Gamma function in which the effective radii and a fixed effective variance are chosen. At each atmospheric layer, the total number of particles is derived from the layer water content for a given effective radius. For ice

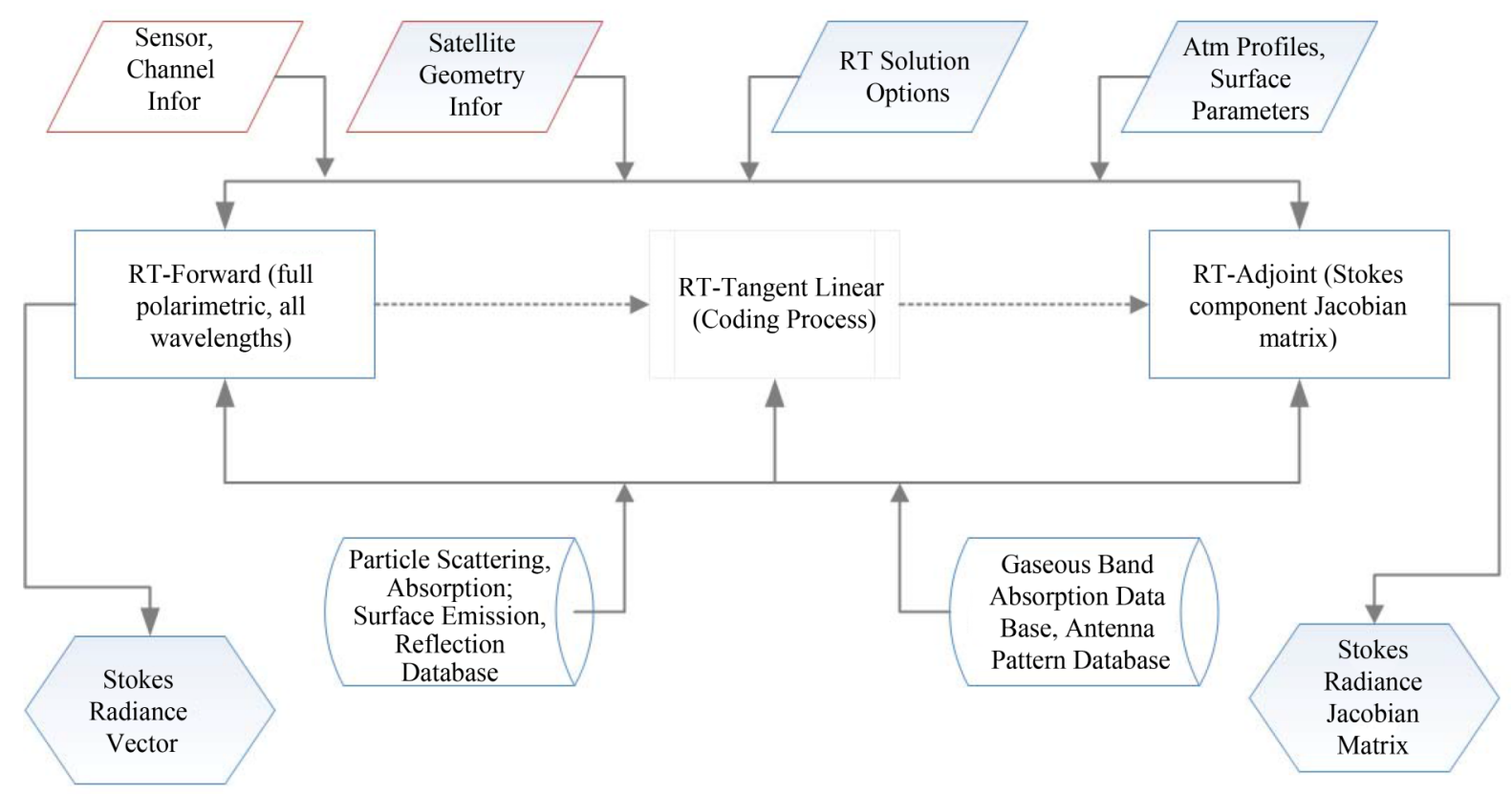

Fig. 1. ARMS, for fast and accurate calculations of satellite radiances and Jacobians at the top of atmospheres. 
clouds, the scattering by particles similar to or larger than the wavelength of light is treated using a T-matrix (Bi and Yang, 2017) and is stored as a wavelength-consistent scattering database. The T-matrix is also used for computing the scattering parameters of aerosol particles.

The water-surface emissivity at microwave wavelengths is computed with a parameterized two-scale roughness model (Weng, 2017), which is called the Fast Emissivity Model, or FASTEM (Liu et al., 2011). In the two-scale model, the largescale roughness is simulated from many small facets whose slopes follow the normal and isotropic distribution (Cox and Munk, 1954). The emission and reflection for each facet is approximated as a specular surface and the reflection at the viewing angle is calculated from the Fresnel formula. The effects of wave shadowing and surface reflection are taken into consideration. The small-scale capillary waves have a wavelength comparable to the radiation wavelength and their reflection is modeled through Bragg scattering. At the infrared wavelength, the water emissivity is simulated by the numerous small facets having a Cox-Munk slope distribution (Wu and Smith, 1997).

Land emissivity at the microwave frequency was developed earlier by Weng et al., (2001). It has a two-layer medium with the top layer having the scatters related to vegetation, or snow, and with the bottom soil layer. The soil reflectivities are first computed from the Fresnel formula and are then weighted with roughness and cross polarization factors. The radiation from volumetric scattering of vegetation or snow is derived from a two-stream radiative transfer model (Weng and Grody, 2000). The latest updates in surface roughness in the ARMS land emissivity model result in better simulations of microwave radiances at a wider frequency range (Chen and Weng, 2016). A polarization-dependent soil roughness model (Shi et al., 2005) is being implemented into the ARMS surface emissivity model.

Since the First Workshop on Remote Sensing and Modeling of Surface Properties held in Paris, France in 2006, the assimilation of satellite surface sensitive data in NWP models has been significantly improved. NWP centers have developed their respective strategies from the uses of land emissivity models and land surface products. In RTTOV, a Tool to Estimate Land Surface Emissivities at Microwave frequencies, or TELSEM (Aires et al., 2011) and the Combined ASTER and MODIS Emissivity over Land, or CAMEL (Borbas et al., 2017) at infrared wavelengths are used for simulations. In CRTM, the snow and sea-ice microwave emissivities are derived using satellite brightness temperatures at window channels and are stored as static databases at variable frequencies and viewing angles (Yan et al., 2008; Yan and Weng, 2011). These datasets are used to generate the emissivity spectra according to snow/sea-ice types. The spectrum range covers the frequency ranging from 5 to $200 \mathrm{GHz}$. These emissivity databases are also utilized in ARMS as an alternative solution to the land emissivity.

Currently, a polarized two-stream model (Liu and Weng, 2002) is implemented as a standard solver in ARMS. In addition, the advanced doubling-adding (or ADA) method (Weng and Liu, 2003; Liu and Weng, 2006), the vector double and adding (or VADA) scheme (Evans and Stephens, 1991), a vector discrete-ordinate radiative transfer model (or VDISORT) (Weng, 1992, 2017; Schulz et al., 1999) are also considered as alternative solvers in ARMS. These advanced solvers are designed for more accurate simulations in UV to visible wavelengths where scattering from molecules and aerosols can exhibit high anisotropic behaviors and require more streams in radiative transfer simulations. Users can also plug their own solvers into ARMS while taking full advantage of the system's surface and atmospheric optical calculations.

\section{Preliminary results from ARMS simulations on FengYun satellite observations}

Since ARMS is mainly being developed for accelerating the use of Chinese FengYun (FY) satellite data in NWP systems, the transmittance models for the FY instruments must be first generated. In addition, we also take some of the optical thickness databases from CRTM and convert them into ARMS format. So far, the optical databases are available for the instruments listed in Table 1. An example of a simulated brightness temperature spectrum of FY-3D's HIRAS (Hyperspectral Infrared Atmospheric Sounder) and its comparison with observation is shown in Fig. 2. The overall biases at longwave and midwave bands are small, while that in the shortwave band between 2200 and $2400 \mathrm{~cm}^{-1}$ is large. This may be due to the non-local thermal equilibrium effects, which are not included in the current ARMS daytime simulation. The simulation accuracy of ARMS has been intercompared with that from CRTM, which has been widely used by the community for satellite data assimilation. The biases and RMSEs of both models with respect to LBLRTM are documented elsewhere (Kan et al., 2019). Here, we are more concerned about the relative biases between the two models. For HIRAS long-wave to midwave bands, the difference is less than $0.0002 \%$ (Fig. 3). For mid-wave sounding instruments, the brightness temperature is accurate to the third decimal place and the computational speed is much faster, compared to both RTTOV and CRTM (figure omitted). The gain in computational time is probably more attributable to the use of a faster polarization two-stream radiative transfer solver.

\section{The pathway for transitioning ARMS into operation and the plan for future development}

Since the ARMS framework is designed with flexible interfaces with different NWP systems, it is being tested in two satellite data assimilation systems running in the community. The Global/Regional Assimilation and Prediction System 
Table 1. Instrument-specific fast transmittance model developed for ARMS applications (instruments in italic are onboard polarorbiting satellite systems and the rest are onboard geostationary satellite systems).

\begin{tabular}{|c|c|c|c|}
\hline US & Europe & China & Japan \\
\hline CrIS & IASI & HIRAS & AMSR-2 \\
\hline ATMS & AMSU & GIIRS & \\
\hline AMSU & MHS & MWTS & \\
\hline MHS & AVHRR & MWHS & \\
\hline HIRS & & MWRI & \\
\hline MSU & & MERSI & \\
\hline SSU & & MERSI-II & \\
\hline \multicolumn{4}{|l|}{ AVHRR } \\
\hline \multicolumn{4}{|l|}{ MODIS } \\
\hline \multicolumn{4}{|l|}{ AMSR-E } \\
\hline \multicolumn{4}{|l|}{ AIRS } \\
\hline \multicolumn{4}{|l|}{ HSB } \\
\hline \multicolumn{4}{|l|}{$\mathrm{SSM} / \mathrm{I}$} \\
\hline \multicolumn{4}{|l|}{$\mathrm{SSM} / \mathrm{T} 1$} \\
\hline \multicolumn{4}{|l|}{$\mathrm{SSM} / \mathrm{T} 2$} \\
\hline \multicolumn{4}{|l|}{ SSMIS } \\
\hline$A B I$ & SEVERI & $A G R I$ & $A H I$ \\
\hline GOES-Imager & & GIIRS & \\
\hline GOES-Sounder & & GOMIS & \\
\hline
\end{tabular}
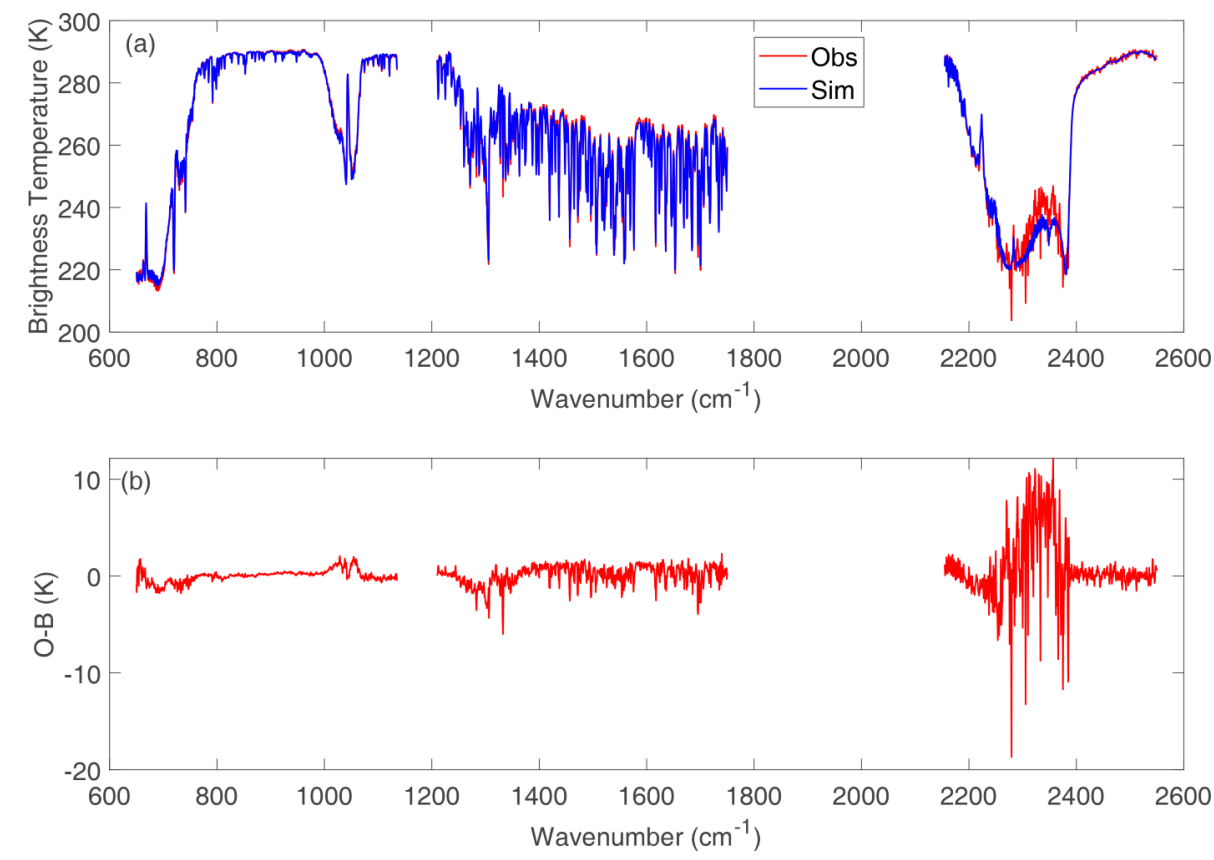

Fig. 2. (a) Simulated brightness temperature spectrum for the HIRAS instrument onboard the FY-3D satellite and comparison with (b) the difference between observations $(\mathrm{O})$ and simulations (B).

(GRAPES) was developed, and is commonly used for, both operation and research (Yu et al., 2019). As a core component, GRAPES' four-dimensional variation (4D-Var) data assimilation system was developed, and is used for, satellite data assimilation. The satellite observation operator used in GRAPES 4D-Var is RTTOV, having been updated for assimilating satellite data including those from AMSU-A/MHS, ATMS, CrIS, MWTS/MWHS, AIRS, IASI, and GIIRS. Since RTTOV is maintained and updated by EUMETSAT NWP SAF, which is led by the UK Met Office with contribution from Meteo France, any request for assimilating the new data stream through RTTOV has to be closely coordinated with those organizations. With ARMS, we are developing a software interface with GRAPES 4D-Var and have begun various data assimilation experiments. 

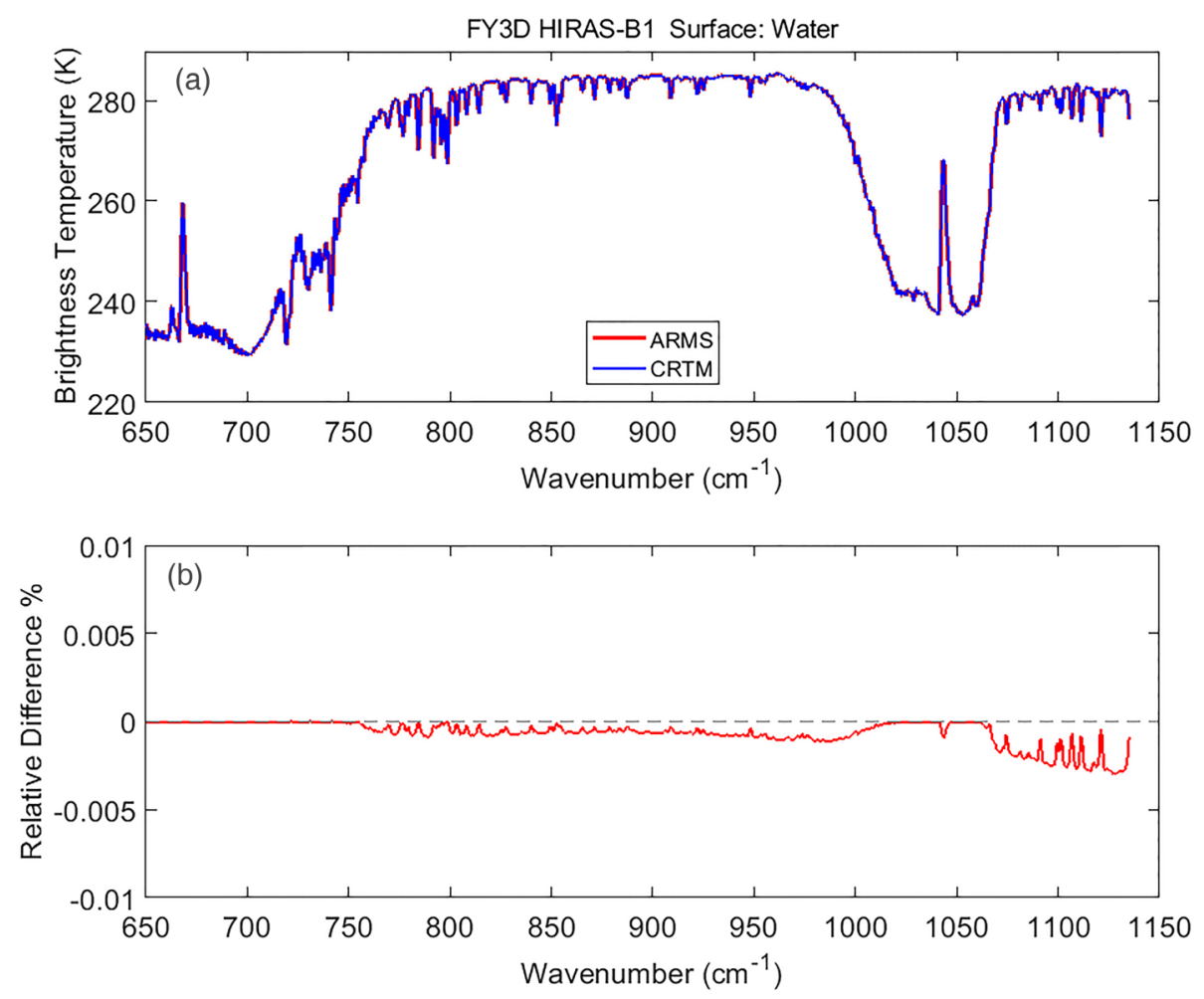

Fig. 3. (a) Simulation accuracy of HIRAS from ARMS and (b) difference between CRTM and ARMS.

The NOAA data assimilation system takes a three-dimensional variational (3D-Var) approach and has inherited the Gridpoint Statistical Interpolation (GSI) analysis system (Wu et al., 2002). GSI is a community data assimilation system with a comprehensive user guide and can be installed and compiled on various computer systems. CRTM is part of GSI and supports a large number of sensors onboard historical GOES/POES satellites. Unfortunately, CRTM does not support the new generation of FengYun satellites. Thus, we are now integrating ARMS into GSI so that all its users can perform assimilations of satellite data from global observing systems.

While ARMS is now ready for assimilating radiance data from satellite sounding instruments, it requires more scientific refinements to many modules. The near-term priority is to improve the simulation accuracy of satellite sounding instruments. In clear conditions, atmospheric optical models should consider trace gas components as NWP models start using more satellite measurements that are sensitive to them. For upper-air microwave sounding channels having weighting functions peaking above $40 \mathrm{~km}$, Zeeman splitting absorption will be considered in ARMS (Han et al., 2007). At infrared wavelengths near $4 \mu \mathrm{m}$, absorbing aerosols can have significant effects on radiance simulation. Volcanic gases and aerosols can affect the radiance simulation at longwave infrared regions and should be fully considered in the transmittance calculation. At UV and visible wavelengths, the atmospheric gases also have scattering and create strong polarization. These features must also be accurately simulated in fast radiative transfer models. ARMS will include Rayleigh scattering of molecules and rotational Raman scattering when the UV hyperspectral data are ready for NWP data assimilation.

The gaps in our knowledge regarding surface emissivities must be filled. In ocean emissivity models, the water dielectric constant affected by salinity was updated earlier in FASTEM (Liu et al., 2011), but has been found to be incomplete at lower frequencies (Lawrence et al., 2017). Sea-ice emissivity is also limited. Over land, an infrared emissivity model has not yet been fully developed for operational fast and accurate radiative transfer models.

Acknowledgements. We would like to thank the support of the National Key Research and Development Program of China "Development of Meteorological Satellite Remote Sensing Technology and Platform for Global Monitoring, Assessments and Applications under the funding code of 2018YFC1506500".

\section{REFERENCES}

Aires, F., C. Prigent, F. Bernardo, C. Jiménez, R. Saunders, and P. Brunel, 2011: A tool to estimate land-surface emissivities at microwave frequencies (TELSEM) for use in numerical weather prediction. Quart. J. Roy. Meteorol. Soc., 137, 690-699, https://doi.org/10.1002/qj.803.

Bi, L., and P. Yang, 2017: Improved ice particle optical property simulations in the ultraviolet to far-infrared regime. Journal of Quantitat- 
ive Spectroscopy and Radiative Transfer, 189, 228-237, https://doi.org/10.1016/j.jqsrt.2016.12.007.

Borbas, E., G. Hulley, W. Feltz, R. Knuteson, and S. Hook, 2017: Combined ASTER and MODIS Emissivity over Land (CAMEL) product. Proc. 21 st Int. TOV Study Conf., Darmstadt, Germany.

Chen, M., and F. Z. Weng, 2016: Modeling land surface roughness effect on soil microwave emission in community surface emissivity model. IEEE Trans. Geosci. Remote Sens., 54, 1716-1726, https://doi.org/10.1109/TGRS.2015.2487885.

Clough, S. A., M. J. Iacono, and J. L. Moncet, 1992: Line-by-line calculations of atmospheric fluxes and cooling rates: Application to water vapor. J. Geophys. Res., 97, 15 761-15 785, https://doi.org/10.1029/92JD01419.

Cox, C., and W. Munk, 1954: Statistics of the sea surface derived from sun glitter. J. Mar. Res., 13, 198-227.

Evans, K. F., and G. L. Stephens, 1991: A new polarized atmospheric radiative transfer model. Journal of Quantitative Spectroscopy and Radiative Transfer, 46, 413-423, https://doi.org/10.1016/0022-4073(91)90043-P.

Han, Y., F. Z. Weng, Q. H. Liu, and P. van Delst, 2007: A fast radiative transfer model for SSMIS upper atmosphere sounding channels. J. Geophys. Res., 112, D11121, https://doi.org/10.1029/2006JD008208.

Kan, W., P. Dong, and S. Ding, 2019: Validation and inter-comparison of ARMS fast transmittance model for FY-4A GIIRS with RTTOV. J. Quant. Spectrosc. Radiat. Transfer., in press.

Lawrence, H., N. Bormann, A. Geer, and S. English, 2017: Uncertainties in the dielectric constant model for seawater in FASTEM and implications for the cal/val of new microwave instruments. Proc. 21st Int. TOV Study Conf., Darmstadt, Germany, 11/29-12/3.

Le Marshall, J., and Coauthors, 2007: The joint center for satellite data assimilation. Bull. Amer. Meteor. Soc., 88, 329-340, https://doi.org/10.1175/BAMS-88-3-329.

Liu, Q. H., and F. Z. Weng, 2002: A microwave polarimetric two-stream radiative transfer model. J. Atmos. Sci., 59, 2396-2402, https://doi.org/10.1175/1520-0469(2002)059<2396:AMPTSR>2.0.CO;2.

Liu, Q. H., and F. Z. Weng, 2006: Advanced doubling-adding method for radiative transfer in planetary atmospheres. J. Atmos. Sci., 63, 3459-3465, https://doi.org/10.1175/JAS3808.1.

Liu, Q. H., F. Z. Weng, and S. J. English, 2011: An improved fast microwave water emissivity model. IEEE Trans. Geosci. Remote Sens., 49, 1238-1250, https://doi.org/10.1109/TGRS.2010.2064779.

McMillin, L. M., and H. E. Fleming, 1976: Atmospheric transmittance of an absorbing gas: A computationally fast and accurate transmittance model for absorbing gases with constant mixing ratios in inhomogeneous atmospheres. Appl. Opt., 15, 358-363, https://doi.org/10.1364/AO.15.000358.

Rothman, L. S., and Coauthors, 2013: The HITRAN 2012 molecular spectroscopic database. Journal of Quantitative Spectroscopy and Radiative Transfer, 130, 4-50, https://doi.org/10.1016/j.jqsrt.2013.07.002.

Saunders, R., M. Matricardi, and P. Brunel, 1999: An improved fast radiative transfer model for assimilation of satellite radiance observations. Quart. J. Roy. Meteorol. Soc., 125, 1407-1425, https://doi.org/10.1002/qj.1999.49712555615.

Saunders, R., and Coauthors, 2018: An update on the RTTOV fast radiative transfer model (currently at version 12). Geoscientific Model Development, 11, 2717-2737, https://doi.org/10.5194/gmd-11-2717-2018.

Schulz, F. M., K. Stamnes, and F. Weng, 1999: Vdisort: An improved and generalized discrete ordinate method for polarized (vector) radiative transfer. Journal of Quantitative Spectroscopy and Radiative Transfer, 61, 105-122, https://doi.org/10.1016/S00224073(97)00215-X.

Shi, J. C., L. M. Jiang, L. X. Zhang, K. S. Chen, J. P. Wigneron, and A. Chanzy, 2005: A parameterized multifrequency-polarization surface emission model. IEEE Trans. Geosci. Remote Sens., 43, 2831-2841, https://doi.org/10.1109/TGRS.2005.857902.

Weng, F. Z., 1992: A multi-layer discrete-ordinate method for vector radiative transfer in a vertically-inhomogeneous, emitting and scattering atmosphere-I. theory. Journal of Quantitative Spectroscopy and Radiative Transfer, 47, 19-33, https://doi.org/10.1016/ 0022-4073(92)90076-G.

Weng, F. Z., 2007: Advances in radiative transfer modeling in support of satellite data assimilation. J. Atmos. Sci., 64, 3799-3807, https://doi.org/10.1175/2007JAS2112.1.

Weng, F. Z., 2017: Passive Microwave Remote Sensing of the Earth: For Meteorological Applications. Wiley-VCH, 384 pp.

Weng, F. Z., and N. C. Grody, 2000: Retrieval of ice cloud parameters using a microwave imaging radiometer. J. Atmos. Sci., 57, 1069-1081, https://doi.org/10.1175/1520-0469(2000)057<1069:ROICPU>2.0.CO;2.

Weng, F. Z., and Q. H. Liu, 2003: Satellite data assimilation in numerical weather prediction models. Part I: Forward radiative transfer and Jacobian modeling in cloudy atmospheres. J. Atmos. Sci., 60, 2633-2646, https://doi.org/10.1175/1520-0469(2003) 060<2633:SDAINW>2.0.CO;2.

Weng, F. Z., B. H. Yan, and N. C. Grody, 2001: A microwave land emissivity model. J. Geophys. Res., 106, 20 115-20 123, https://doi.org/10.1029/2001JD900019.

Wu, W. S., R. J. Purser, and D. F. Parrish, 2002: Three-dimensional variational analysis with spatially inhomogeneous covariances. Mon. Wea. Rev., 130, 2905-2916, https://doi.org/10.1175/1520-0493(2002)130<2905:TDVAWS>2.0.CO;2.

Wu, X. Q., and W. L. Smith, 1997: Emissivity of rough sea surface for 8-13 $\mu$ m: Modeling and verification. Appl. Opt., 36, 2609-2619, https://doi.org/10.1364/AO.36.002609.

Yan, B. H., and F. Z. Weng, 2011: Effects of microwave desert surface emissivity on AMSU-A data assimilation. IEEE Trans. Geosci. Remote Sens., 49, 1263-1276, https://doi.org/10.1109/TGRS.2010.2091508.

Yan, B. H., F. Z. Weng, and H. Meng, 2008: Retrieval of snow surface microwave emissivity from the advanced microwave sounding unit. J. Geophys. Res., 113, D19206, https://doi.org/10.1029/2007JD009559.

Yu, R. C., Y. Zhang, J. J. Wang, J. Li, H. M. Chen, J. D. Gong, and J. Chen, 2019: Recent progress in numerical atmospheric modeling in China. Adv. Atmos. Sci., 36(9), 938-960, https://doi.org/10.1007/s00376-019-8203-1.

Zou, X., F. Weng, B. Zhang, L. Lin, Z. Qin, and V. Tallapragada, 2013: Impacts of assimilation of ATMS data in HWRF on track and intensity forecasts of 2012 four landfall hurricanes. J. Geophys. Res., 118, 11 558-11 576, https://doi.org/10.1002/2013JD020405. 